\title{
Resposta de duas sessões de natação sobre parâmetros de estresse oxidativo em nadadores
}

\author{
Response of two swimming session on oxidative stress \\ parameters in swimmers
}

\author{
Luciano Acordi da Silva ${ }^{1}$ \\ Luis Gustavo Costa da Rocha \\ Débora Scheffer ${ }^{1}$ \\ Fernanda Schrveizer Soares ${ }^{1}$ \\ Cleber Aurino Pinho ${ }^{1}$ \\ Adriano B. Polizelli ${ }^{1}$ \\ Paulo Cesar Lock Silveira 1 \\ Ricardo Aurino Pinho ${ }^{1}$
}

\footnotetext{
1 Universidade do Extremo Sul Catarinense. Programa de Pós Graduação em Ciências da Saúde. Laboratório de fisiologia e bioquímica do Exercício. Criciúma. Santa Catarina. Brasil.
}

Recebido em 19/06/08 Revisado em 07/11/08 Aprovado em 19/11/08
Resumo - O objetivo do presente estudo foi investigar a resposta aguda de duas sessões de natação sobre parâmetros de estresse oxidativo em indivíduos fisicamente ativos. Doze sujeitos homens $(28 \pm 7$ anos, $1,75 \pm 0.08 \mathrm{~m}, 72.9 \pm 9 \mathrm{~kg})$ com experiência em natação superior a um ano de treinamento realizaram duas sessões de natação, 1 hora por sessão, com intervalo de 12 horas. Foram coletadas amostras de sangue da veia cubital 24 horas antes de prova (C1), imediatamente após a primeira sessão (C2) e segunda sessão (C3) e 24 horas após o término da prova (C4). Foram analisados as atividades da Creatina Quinase (CK), os níveis lipoperoxidação, carbonilação de proteínas e conteúdo total de tióis a atividade da catalase. Os resultados mostram um aumento na atividade da CK (1143.8 $\pm 254.2 \mathrm{U} / \mathrm{L})$ nos níveis de lipoperoxidação (3.01 $0.54 \mathrm{nmol} /$ TBARS/mg proteína) e carbonilação de proteínas (3.01 $\pm 0.54 \mathrm{nmol} / \mathrm{mg}$ de proteína) e uma diminuição no conteúdo total de tióis $(17.09 \pm 3.31 \mathrm{nmol} \mathrm{TNB} / \mathrm{mg}$ proteína) imediatamente após a segunda sessão de natação em comparação a pré-prova $(111.2 \pm 33.2 \mathrm{U} / \mathrm{L} ; 1.68 \pm 0.34 \mathrm{nmol} / \mathrm{TBARS} / \mathrm{mg}$ proteína; $1.68 \pm 0.34$ $\mathrm{nmol} / \mathrm{mg}$ de proteína; $26.8 \pm 3.08 \mathrm{nmol} \mathrm{TNB} / \mathrm{mg}$ de proteína) respectivamente. A atividade da catalase aumentou após as duas sessões (C2; 2.5 $\pm 0.35 \mathrm{U} / \mathrm{mg}$ proteína; $\mathrm{C} 3 ; 2.5 \pm 0.47 \mathrm{U} / \mathrm{mg}$ proteína) em comparação a pré-prova $(\mathrm{C} 1 ; 1.5 \pm 0.35 \mathrm{U} / \mathrm{mg}$ proteína). Em conclusão, somente a segunda sessão de natação alterou os parâmetros de estresse oxidativo.

Palavras-chave: Natação; Estresse oxidativo; Radicais livres.

Abstract - This study investigated the acute effect of two swimming sessions on oxidative stress markers in trained individuals. Twelve male volunteers, students from UNESC (Universidade do Extremo Sul Catarinense, Criciuma, Santa Catarina, Brazil), with a mean age of $28 \pm$ 7 years, initial weight of $72.9 \pm 9 \mathrm{~kg}$ and height of $1.75 \pm 0.08 \mathrm{~cm}$, participated in the study. Blood samples were collected 24 hours before the sessions, immediately after the first and second session, and 24 hours after the end of the sessions. Aliquots were washed, red blood cells were lysed and plasma samples were stored at $-80 \circ \mathrm{C}$ until the time of the biochemical assays. Creatine kinase (CK) activity, lipid peroxidation level, protein carbonylation, thiol content and catalase activity were determined. The results showed a significant increase in CK, lipoperoxidation and protein carbonylation and a decrease in thiol content after the second swimming session $(p<0.05)$ compared to pre-swimming levels. Catalase activity increased after the first and second swimming sessions. The main finding of the present study was that only the second swimming session resulted in oxidative stress.

Key words: Swimming; Oxidative stress; Free radicals. 


\section{INTRODUÇÃO}

Nos últimos anos, diversos estudos demonstram a influência do exercício físico sobre a produção das Espécies Reativas de Oxigênio (ERO) em tecidos biológicos ${ }^{1-3}$. Conforme Satre et al. ${ }^{4}$, o exercício físico promove o estresse oxidativo somente quando é exaustivo. Então, é importante distinguir entre o exercício não exaustivo, o qual não acompanha o estresse oxidativo, e o exercício exaustivo o qual causa estresse oxidativo.

Vários mecanismos estão envolvidos na produção de ERO durante o exercício. A maioria dos oxidantes produzidos durantes às contrações musculares são devido ao aumento no consumo de $\mathrm{O}_{2}$ na cadeia respiratória ${ }^{5}$. Contudo, outros mecanismos de produção das ERO durante o exercício também são importantes, como a ativação da xantina oxidase, alteração da homeostase de cálcio, isquemia e reperfusão, ativação de células inflamatórias ${ }^{6-8}$

Durante o exercício, o consumo de $\mathrm{O}_{2}$ para produção aeróbica de ATP pode aumentar de 10 a 20 vezes em relação aos níveis de repouso ${ }^{9}$,causando um aumento concomitante na produção das ERO. Quando o aumento das ERO excede a capacidade de defesa antioxidante das células, pode causar dano na estrutura do DNA, oxidar proteínas, lipídios e carboidratos, caracterizando o fenômeno celular denominado de estresse oxidativo.

Vários estudos em humanos têm delineado os efeitos do exercício e do treinamento físico sobre parâmetros estresse oxidativo-12. Embora a literatura seja vasta neste sentido, faltam estudos que esclareçam a resposta de duas sessões de exercícios exaustivos de natação, sem um tempo de recuperação adequado.

Neste sentido, a proposta do presente estudo foi investigar o efeito agudo de duas sessões (1hora duração cada sessão) de exercício exaustivo de natação sobre marcadores de estresse oxidativo em sujeitos fisicamente ativos.

\section{PROCEDIMENTOS METODOLÓGICOS}

Participaram do estudo 12 (doze) indivíduos voluntários, não atletas, do sexo masculino, com idade média de $28 \pm 7$ anos, com experiência mínima na prática em natação de 24 meses (Tabela 1). Foram excluídos do estudo os sujeitos que utilizavam algum tipo de medicação, suplementos nutricionais, que estavam realizando algum programa de treinamento de exercício físico paralelo ou nos últimos seis meses, fumantes que apresentassem histórico de lesão muscular ou portadores de alguma doença que pudesse comprometer os resultados ou ser agravada pela prática do exercício físico. Todos os participantes preencheram o Termo de Consentimento Informado, que foi aprovado pelo comitê de ética local, conforme parecer n ${ }^{\circ}$ 649/2007.

Tabela 1. Características antropométricas e fisiológicas.

\begin{tabular}{lccccc}
\hline & A1 & A2 & A3 & A4 & A5 \\
\hline $\begin{array}{l}\text { Idade } \\
\text { (anos) }\end{array}$ & $28 \pm 7$ & & & & \\
Altura (m) & $1,75 \pm 0,08$ & & & & \\
Peso (Kg) & $72,9 \pm 9$ & $72,2 \pm 8$ & $71,5 \pm 9$ & $71,3 \pm 9$ & \\
$\begin{array}{l}\text { Freqüência } \\
\text { Cardíaca }\end{array}$ & $64 \pm 8$ & $94 \pm 19$ & $129 \pm 15$ & $95 \pm 21$ & $134 \pm 12$ \\
$\begin{array}{l}\text { (Bpm) } \\
\text { SpO }\end{array}$ & & & & & \\
(mmHg) & & $98,1 \pm 0,7$ & $96,3 \pm 1,6$ & $97 \pm 2,2$ & $94,3 \pm 4,7$ \\
Distancia (m) & & & $2843 \pm 227$ & & $2844 \pm 387$ \\
\hline
\end{tabular}

Os indivíduos foram mensurados vinte quatro horas antes da prova (A1), imediatamente antes da $1^{\circ}$ sessão de nado (A2), logo depois da $1^{\circ}$ sessão de nado (A3), imediatamente antes da $2^{\circ}$ sessão de nado (A4) e logo depois da $2^{\circ}$ sessão de nado (A5). Todos os resultados foram expressos em média e erro padrão médio.

Protocolo do treinamento físico: Os indivíduos praticaram os treinamentos de natação no período da noite (22:00 as 23:30h), sessenta minutos por dia, três a quatro vezes por semana, em piscina coberta de 25 metros, com a temperatura da água em $28^{\circ}$ graus. A distância diária percorrida no treino foi entre $2000 \mathrm{~m}$ e $2500 \mathrm{~m}$.

Prova de natação: $O$ estudo foi realizado na Universidade do Extremo Sul Catarinense durante o $2^{\circ}$ Desafio Nadando Ainda Mais Rápido. $\mathrm{O}$ desafio consistiu em realizar um revezamento com 12 atletas por equipe tendo duração de 24 horas. Cada atleta nadou dois percursos com 01 hora de duração cada. O intervalo entre os percursos foi de 12 horas. Durante o período de repouso os indivíduos permaneceram numa sala apropriada na própria instituição. Durante o desafio de natação não foi utilizado nenhum suplemento, medicamento ou algum tipo de tratamento que pudesse acelerar a recuperação dos nadadores ou interferir nos resultados.

Controle alimentar: Durante o ultimo mês de treinamento os indivíduos receberam uma dieta nutricional balanceada e individualizada, contendo $55 \%$ a $65 \%$ do valor calórico total (VCT) carboidrato, $15 \%$ de proteína e $25 \%$ a $35 \%$ de lipídios. $\mathrm{Na}$ semana que antecede o evento, os atletas receberam orientações nutricionais a fim de garantir uma 
elevação no aporte de carboidratos. A manipulação dietética consistiu em uma redução nos três primeiros dias para 50\% do VCT de carboidrato, e nos quatro dias seguintes a dieta possuía em torno de $70 \%$ do VCT de carboidrato.

Coleta de sangue: As coletas aconteceram vinte e quatro horas antes da prova (C1), logo após a primeira sessão (C2), após a segunda sessão (C3) e vinte quatro horas após o término da prova (C4). Foram coletados $15 \mathrm{~mL}$ de sangue da veia cubital do braço direito. $\mathrm{O}$ sangue foi coletado em tubos de vacutainer heparinizados e as frações (eritrócitos e plasma) foram separadas e estocadas imediatamente em freezer $-80^{\circ} \mathrm{C}$ para posterior análises.

\section{Parâmetro de performance}

A performance foi avaliada pela maior distância percorrida durante as duas sessões de uma hora de nado continuamente. Os nadadores foram encorajados verbalmente a realizarem a maior distância durante os dois períodos.

\section{Ensaios bioquímicos}

Creatina Quinase (CK): Foi determinada em plasma com auxílio de kit específico fornecido pela Labtest Diagnóstica SA., seguindo as orientações técnicas observada na bula do referido kit.

Espécies reativas ao ácido Tiobarbitúrico (TBARS): Foi determinada em plasma como marcador de lipoperoxidação pela formação de substâncias reativas ao aquecimento do ácido tiobarbitúrico medido espectrofotometricamente (532nm), conforme descrito ${ }^{13}$.

Carbonilação de proteínas: Os danos oxidativos em proteínas foram determinados em plasma mensurados pela determinação de grupos carbonils, baseados na reação com dinitrofenilhidrazina como previamente descrito ${ }^{14}$. O conteúdo de carbonil foi determinado espectrofotometricamente em 370nm usando um coeficiente 22.0000 Molar $^{-1}$.

Tióis totais: Foi determinada em plasma numa reação dos grupos tióis com 5,5'ditióbis (2 acido nitro-benzóico) (DTNB), gerando um derivado de coloração amarela e lido espectrofotometricamente a $412 \mathrm{~nm}^{15}$.

Catalase (CAT): $\mathrm{A}$ atividade da catalase (CAT) foi determinada nos eritrócitos pela taxa de decaimento do peróxido de hidrogênio lido em espectofotômetricamente a $240 \mathrm{~nm}^{16}$.

Determinação da proteína: A quantidade de proteínas nos ensaios do TBA, CARBONIL, SUFIDRILA e CAT foram mensurados usando a técnica de Lowry et al. ${ }^{17}$.

\section{Tratamento estatístico}

Os dados foram expressos em média e erro padrão médio e analisados estatisticamente pela análise de variância (ANOVA) one-way, seguido pelo teste post hoc Bonferroni. O nível de significância estabelecido para o teste estatístico é de $\mathrm{p}<0,05$. Foi utilizado o SPSS (Statistical Package for the Social Sciences) versão 15.0 como pacote estatístico.

\section{RESULTADOS}

Performance: Não houve diferença significativa na distância percorrida pelos nadadores entre a primeira $(2843 \pm 227 \mathrm{~m})$ e a segunda $(2844 \pm 387 \mathrm{~m})$ sessão de nado.

Creatina Kinase (CK): Os resultados (Tabela 2) demonstram um aumento na atividade da CK (plasma) logo após a segunda sessão de exercício $(1143,8 \pm 254,2 \mathrm{U} / \mathrm{L})$ e vinte quatro horas após o término da prova $(795,9 \pm 208,1 \mathrm{U} / \mathrm{L})$, quando comparado à pré-prova $(111,2 \pm 33,2 \mathrm{U} / \mathrm{L})$.

Tabela 2. Atividade da Creatina Quinase (CK) e Catalase (CAT).

\begin{tabular}{lcccc}
\hline & C1 & C2 & C3 & C4 \\
\hline CK (U/L) & $114,2 \pm 33$ & $348,7 \pm 162$ & $1143,8 \pm 254^{*}$ & $795,9 \pm 208^{*}$ \\
$\begin{array}{l}\text { CAT U/mg } \\
\text { proteína }\end{array}$ & $1,5 \pm 0,35$ & $2,5 \pm 0,48^{*}$ & $2,5 \pm 0,47^{*}$ & $2,1 \pm 0,35$ \\
\hline
\end{tabular}

As coletas foram mensuradas e descritas de acordo com materiais e métodos. Os valores são apresentados em Média土EPM e os resultados de CK foram expressos em (U/L) e a atividade da CAT (U/mg de prieína). A diferença significativa encontrada em relação à primeira coleta $(*)$ foi de $p<0,05$.

Lipoperoxidação: Na Figura 1, os resultados mostram um aumento nos níveis de lipoperoxidação no plasma, logo após a segunda sessão de

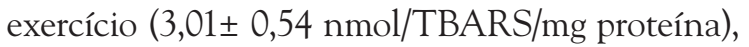
quando comparado à pré-prova $(1,68 \pm 0,34 \mathrm{nmol} /$ TBARS/mg proteína).

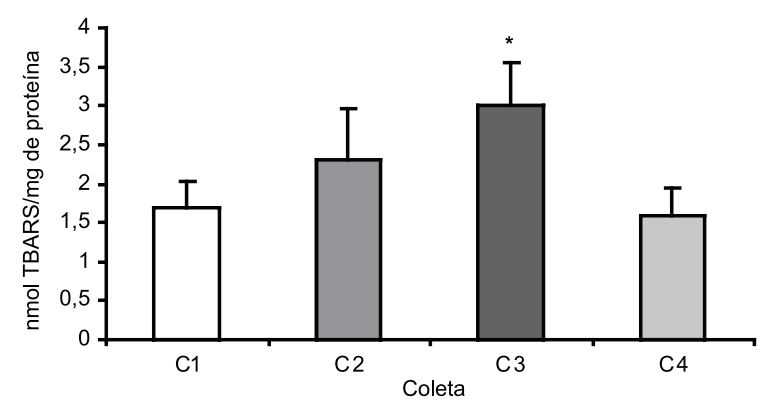

Figura 1. As Substâncias Reativas ao Ácido Tiobarbitúrico (TBARS) foram determinadas de acordo com materiais e métodos. Os valores são apresentados em Média土EPM e os resultados foram expressos em (nmol TBARS/mg de proteína). A diferença significativa encontrada em relação à primeira coleta $(*)$ foi de $p<0,05$. 
Carbonilação de proteína (CP): Os resultados mostram (Figura 2) um aumento na CP no plasma, após a segunda sessão de exercício $(3,01 \pm 0,54$ nmol/mg de proteína), em comparação à pré-prova $(1,68 \pm 0,34 \mathrm{nmol} / \mathrm{mg}$ de proteína).

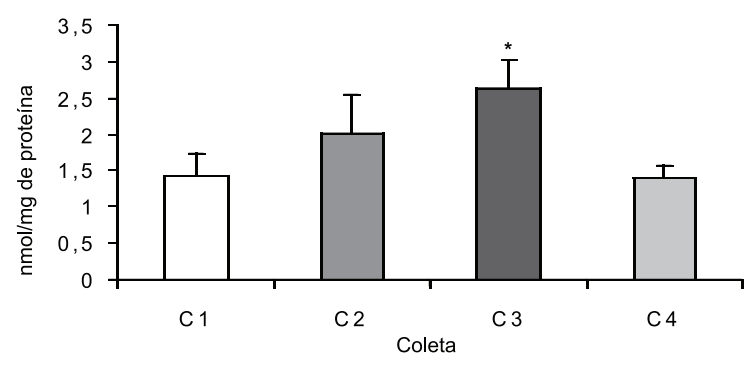

Figura 2. A carbonilação de proteína foi determinada de acordo com materiais e métodos. Os valores são apresentados em Média土EPM e os resultados foram expressos em (nmol/mg de proteína). A diferença significativa foi encontrada em relação à primeira coleta $(*)$ foi de $\mathrm{p}<0,05$.

Total de Tióis(TT): Na Figura 3, foi observada uma diminuição nas proteínas não oxidadas (TT), logo após a segunda sessão de exercício $(17,09 \pm 3,31$ nmol TNB/mg proteína), quando comparado à préprova $(26,8 \pm 3,08 \mathrm{nmol} \mathrm{TNB} / \mathrm{mg}$ de proteína).

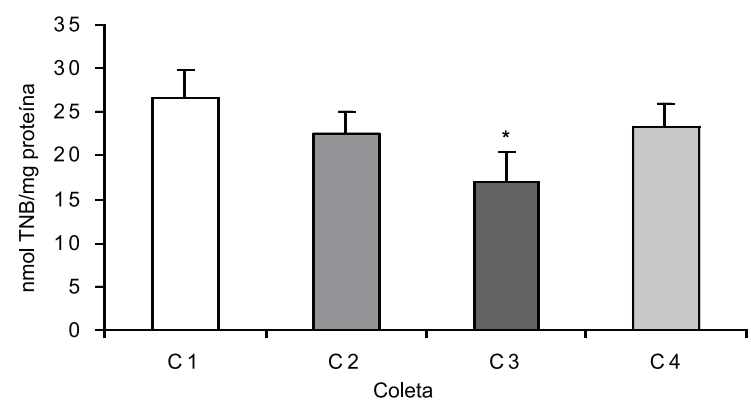

Figura 3. O conteúdo TT foi determinado de acordo com materiais e métodos. Os valores são apresentados em Média土EPM e os resultados foram expressos em (nmol/mg proteína). A diferença significativa foi encontrada em relação à primeira coleta $\left(^{*}\right)$ foi de $p<0,05$.

Catalase (CAT): Na Tabela 2, os resultados mostram um aumento na atividade da CAT logo após a primeira e a segunda sessão de exercício (2,5 $\pm 0,35 \mathrm{U} / \mathrm{mg}$ proteína; $2,5 \pm 0,47 \mathrm{U} / \mathrm{mg}$ proteína) em comparação à pré-prova $(1,5 \pm 0,35 \mathrm{U} / \mathrm{mg}$ proteína).

\section{DISCUSSÃO}

Mudanças nos marcadores de Estresse Oxidativo (EO) em nadadores acontecem depois de exercícios de natação $0^{11,12}$. Estudos têm delineado o efeito do exercício crônico no estado redox e nas defesas antioxidantes em nadadores ${ }^{10,18,19}$. Diferentemente destes trabalhos, nosso estudo demonstrou a res- posta aguda de duas sessões de natação de longa duração (60 min de nado competitivo +12 horas de repouso $+60 \mathrm{~min}$ de nado competitivo), sobre parâmetros de estresse oxidativo.

Nossos resultados mostraram aumento no dano muscular (CK), nos marcadores de dano oxidativo (TBARS e CP) e uma diminuição no conteúdo das proteínas não oxidadas (TT), somente após a segunda sessão de exercício. A atividade da catalase aumentou após a primeira e a segunda sessão. Apontamos ainda que uma sessão de natação não causa estresse oxidativo.

Uma quantia mínima de ERO é necessária para contração muscular ${ }^{20,21}$. Todavia, quando esta produção de ERO excede a capacidade antioxidante de defesa, ocorre o que chamamos de EO. O EO pode estar associado com a fadiga muscular durante as contrações e após os exercícios intensos ${ }^{22,23}$. Uma das hipóteses que explica o efeito das ERO na fadiga muscular está relacionada às alterações no funcionamento mitocondrial induzido pelas $\mathrm{ERO}^{24}$. Exercícios intensos levam a uma grande produção de ERO o que pode danificar lipídeos, proteínas e DNA. Adicionalmente, pode induzir alterações no mtDNA e provocar danos nos complexos da cadeia transportadora de elétrons o que, em consequência, diminui a transferência de elétrons e a formação de ATP'. Este fenômeno pode aumentar a participação do metabolismo anaeróbio na produção de energia e comprometer a performance ${ }^{24}$. Nós hipotetizamos que a primeira sessão de natação alteraria os marcadores de dano oxidativo, interferindo na performance dos nadadores na segunda sessão de exercício. Entretanto, isto não foi observado e em ambos os percursos os nadadores percorreram distâncias similares (Tabela 1).

Como marcador de dano muscular, avaliamos a atividade da CK plasmática. A CK no músculo esquelético catalisa a reação reversível da quebra da fosfocreatina. $\mathrm{O}$ aumento na atividade da CK no plasma tem sido usado como marcador de lesão da musculatura esquelética após os exercícios ${ }^{7,25}$. O presente estudo demonstrou o pico da CK logo após a segunda sessão de exercício (Tabela 2). Este resultado difere-se de outros estudos ${ }^{25,26}$ que observaram o pico de CK, 24 horas após uma sessão de exercício. Outros trabalhos ainda ${ }^{7,27}$, demonstram um pico de CK 48 horas após o exercício. A diferença entre esses resultados pode estar associada aos diferentes protocolos de exercícios utilizados. Nos estudos supracitados, as atividades foram maratona, ultramaratona ou ainda, exercícios que envolviam predominantemente contrações excêntricas. $\mathrm{Ou}$ - 
tros fatores que podem influenciar as concentrações de CK é o percentual de massa muscular ${ }^{25}$, que em homens é maior quando comparado ao das mulheres e os níveis de estrogênio, que nas mulheres podem aumentar a habilidade sarcoplasmática, reduzindo o fluxo da $\mathrm{CK}$ de dentro para fora da célula muscular ${ }^{28}$. Especificadamente, na modalidade de natação, acreditamos que a diminuição do atrito muscular que a água proporciona em relação ao peso corporal dos indivíduos, pode fazer com que uma sessão de natação não seja suficiente para causar significativo dano muscular.

Como parâmetros de dano oxidativo, nós avaliamos a lipoperoxidação de lipídeos e a carbonilação de proteínas. As ERO podem oxidar lipídeos de membranas em particular ácidos graxos livres polisaturados, sendo que estes constituem as membranas celulares $^{2,3,27}$. Nossos resultados estão de acordo com outros trabalhos ${ }^{10}$, demonstrando aumento nos níveis do TBARS após o exercício (Figura 1). Especificamente em nosso estudo, tanto a lipoperoxidação quanto a carbonilação de proteínas aumentaram após a segunda sessão de natação (Figura 2).

As ERO produzidas durante os exercícios podem oxidar as estruturas das proteínas e lipídeos ${ }^{22}$. Então, essa oxidação pode fazer com que os aminoácidos das proteínas sejam desfragmentados ${ }^{9}$. Essas reações conduzem a alterações no funcionamento de várias enzimas ${ }^{18-5}$. A oxidação dos aminoácidos é acompanhada pelo aumento relativo do nível de proteínas carbonis ${ }^{29}$, o qual é usualmente utilizado como marcador de dano oxidativo. É possível que em indivíduos fisicamente ativos e adaptados a este modelo de exercício (treinados em piscina), a produção das ERO induzida pelo exercício seja menor, e a expressão das enzimas antioxidantes induzida pelo treinamento seja maior (esta hipótese precisa ser confirmada), fazendo com que uma sessão de exercício não altere os marcadores de dano oxidativo. Acreditamos, ainda, que outros sistemas de defesas enzimáticos e não enzimáticos possam estar atuando neste processo.

Outro marcador importante da oxidação da proteínas é o conteúdo de tióis total (TT). Esta técnica verifica a quantidade de sufidrilas $(\mathrm{SH})$ não oxidadas, que estão presentes nos aminoácidos ${ }^{15}$. $\mathrm{O}$ grupo $\mathrm{SH}$ pode ser oxidado por radicais livres, comprometendo o funcionamento das proteínas. O sistema de defesa antioxidante regula o estado redox celular dos Tiós, protegendo contra a excessiva oxidação. Nosso trabalho demonstrou que o conteúdo total de tióis diminui somente após a segunda sessão de exercício (Figura 3).
Este resultado corresponde ao aumento da CP e TBARS. No esporte de escalada indor, Magalhães e colaboradores ${ }^{1}$ demonstraram diminuição das proteínas sufidrilas $(\mathrm{SH})$ depois do exercício exaustivo. $\mathrm{O}$ exercício intenso provoca condições isquêmicas musculares que ativam a rota da xantina oxidase, formando ácido úrico. Durante esse processo ocorre aumento na concentração de cálcio intramuscular que ativam proteases, que ao ser oxidadas diminuem o conteúdo de sufidrila ${ }^{30}$. A quantidade de proteínas oxidadas no plasma foi diminuída significativamente depois da segunda sessão de exercício. Isto provavelmente contribui para disfunções celulares como perda da atividade catalítica, estrutural e integridade das membranas celulares, interrompendo rotas fisiológicas importantes. Nossos resultados estão de acordo com outros trabalhos ${ }^{2,3,10}$ que demonstram que o exercício físico aumenta o dano oxidativo (CP e TBARS) e diminui o conteúdo de sufidrila ${ }^{1}$.

A atividade antioxidante enzimática da CAT foi avaliada em eritrócitos. De acordo com os resultados (Tabela 2), observamos um aumento da atividade logo após as duas sessões de exercício. Alguns estudos têm mostrado o aumento na atividade das enzimas antioxidantes, após um período de treinamento físico em humanos ${ }^{11,12}$, porém, outros estudos apontam para uma diminuição ${ }^{10}$. É provável que resultados diferentes encontrados na literatura sejam decorrentes das diferentes técnicas utilizadas, da treinabilidade dos indivíduos ou ainda, quanto ao tipo de amostra escolhida para dosagem. Acreditamos que esta elevação da CAT logo após a primeira sessão, justifique a não alteração nos marcadores de danos oxidativos. Porém, após a segunda sessão, o aumento da atividade da CAT não foi suficiente para proteger o dano oxidativo.

\section{CONCLUSÃO}

Uma sessão de exercício exaustivo de natação não é suficiente para provocar alterações significativas nos marcadores de danos oxidativos. Entretanto, duas sessões de natação sem um tempo de recuperação adequado induzem a dano muscular e estresse oxidativo.

\section{REFERÊNCIAS BIBLIOGRÁFICAS}

1. Magalhães J, Ferreira R, Marques F, Oliveira E, Soares J, Ascensão. Indoor climbing elicits plasma oxidative stress. Med Sci Sports Exerc 2007;39(6):955-963.

2. Pinho RA, Andrades ME, Oliveira MR, Pirola AC, Zago MS, Silveira PC, Dal-Pizzol F, Moreira JC. Imba- 
lance in SOD/CAT activities in rat skeletal muscles submitted to treadmill training exercise. Cell Biol Int 2006;30(10):848-853.

3. Alessio HM, Hagerman AE, Fulkerson BK, Ambrose J, Rice RE, Wiley RL. Generation of reactive oxygen species after exhaustive aerobic and isometric exercise. Med Sci Sports Exerc 2000;32(9):1576-1581.

4. Sastre J, Asensi M, Gascó E, Pallardó FV, Ferrero JA, Furukawa et al. Exhaustive physical exercise causes oxidation of glutathione status in blood: Prevention by antioxidant administration. Am J Physiol 1992;32(5):992-995.

5. Powers SK, Ji LL, Leeuwenburgh C. Exercise traininginduced alterations in skeletal muscle antioxidant capacity: a brief review. Med Sci Sports Exerc 1999;31(7):987-997.

6. Childs A, Jacobs C, Kaminski T, Halliwell B, Leeuwenburgh C. Supplementation with vitamin $\mathrm{C}$ and $\mathrm{N}$-acetyl-cysteine increases oxidative stress in humans after an acute muscle injury induced by eccentric exercise. Free Radic Biol Med 2001;31(6):745-753.

7. Goldfarb AH, Bloomer RJ, McKenzie MJ. Combined antioxidant treatment effects on blood oxidative stress after eccentric exercise. Med Sci Sports Exerc 2005;37(2):234-239.

8. Hessel E, Haberland A, Muller M,Lerche D, Schimke I. Oxygen radical generation of neutrophils: a reason for oxidative stress during marathon running? Clin Chim Acta 2000;1(2):145-156.

9. Finaud J, Scislowski V, Lac G, Durand D, Vidalin H, Robert A. Antioxidant status and oxidative stress in professional rugby players: evolution throughout a season. Int J Sports Med 2006;27(2):87-93.

10. Gougoura S, Nikolaidis MG, Kostaropoulos IA, Jamurtas AZ, Koukoulis G, Kouretas D. Increased oxidative stress indices in the blood of child swimmers. Eur J Appl Physiol 2007;100(2):235-239.

11. Aguiar-Silva RH, Cintra BB, Milani S, Moraes TP, Tsuji H. Estado antioxidante do Sangue como indicador da eficiência do treinamento em nadadores. Rev Brás Ciên Mov 2002;10(3):7-11.

12. Siems WG, Brenke R, Sommerburg O, Grune T. Improved antioxidative protection in winter swimmers. Q J Med 1999;92(4):193-198.

13. Draper HH, Hadley M. Malondialdehyde determination as index of lipid peroxidation. Methods Enzymol 1990;186(1):421-431.

14. Levine RL, Garland D, Oliver CN, Amici A, Climent I, Lenz AG, Ahn BW, Stadtman ER. Determination of carbonyl content in oxidatively modified proteins. Meth Enzymol 1990;186(1):464-478.

15. Aksenov MY, Markesbery WR. Changes in thiol content and expression of glutathione redox system genes in the hippocampus and cerebellum in Alzheimer's disease. Neurosci Lett 2001;302(2):141-145.

16. Aebi H. Catalase in vitro. Meth Enzymol 1984;105(1):121-126

17. Lowry OH, Rosebough NG, Farr AL, Randall RJ. Protein measurement with the folin phenol reagent. J Biol Chem 1951;193(1):265-275.
18. Cavas L, Tarhan L. Effects of viatamin-mineral supplementation on cardiac marker and radical scavenging enzymes, and MDA levels in young swimmers. Int J Sport Nutr Exerc Metab 2004;14(2):133-146.

19. Santo-Silva A, Rebelo MI, Castro EMB, Belo L, Guerra A, Rego C, Quintanilha A. Leukocyte activation, eruthrocyte damage, lipid profile and oxidative stress imposed by high competition physical exercise in adolescent. Clin Chim Acta 2001;306(1):119-126.

20. Reid MB.Invited review: redox modulation of the skeletal muscle contraction: what we know and what we don't. J Appl Physiol 2001;90(2):724-31.

21. Andrade FH, Reid MB, Allen DG, Westerblad H. Effect of hydrogen peroxide and dithiothreitol on contractile function of single skeletal muscle fibres from the mouse. J Physiol 1998;509(2):565-75.

22. Cooper CE, Vollaard NBJ, Choueiri T, Wilson MT. Exercise, free radical and oxidative stress. Biochem Soc Trans 2002;30(2):280-285.

23. Dawson B, Henry GJ, Goodman C, Gillam I, Beiby JR,Ching S, et al. Effect of vitamin C and E supplementation on biochemical and ultrastructural indices of muscle damage after $21 \mathrm{Km}$ run. Int J Sports Med 2002;23(1):10-15.

24. Reid MB, Haack KE, Franchek KM, Valberg PA, Kobzik L, West MS. Reactive oxygen in skeletal muscle I: intracellular oxidants kinetics and fatigue in vitro. J Appl Physiol 1992;73(5):1797-1804.

25. Mastaloudis A, Traber MG, Carstensen K, Widrick JJ. Antioxidants did not prevent muscle damage in response to an ultramarathon run. Med Sci Sports Exerc 2006;38(1):72-80.

26. Armstrong R. Muscle damage and endurance events. Sports Med 1986;3 (5):370-381.

27. Bloomer RJ, Goldfarb AH, McKenzie MJ, You T, Nguyen L. Effects of antioxidant therapy in women exposed to eccentric exercise. Int J Sport Nutr Exerc Metab 2004;14 (4):377-388.

28. Tidus P. Estrogen and gender effects on muscle damage, inflammation, and stress oxidative. Can J Appl Physiol 2000;25 (4):274-287.

29. Packer L. Oxidants, antioxidants nutrients and the athlete. J Sports Sci 1997;15(3):353-363.

30. Hellsten Y. The role of xanthine oxidase in exercise. In: Sen CK, Parker L, Hanninen O. editors. Handbook of oxidants and antioxidants in exercise. Basel: Elsevier Science BV; 2000.p.153-176.
Endereço para correspondência
Laboratório de Fisiologia e Bioquímica do Exercício/UNESC
Av. Universitária, 1105 - Bairro Universitário 88806-000 - Criciúma - SC - Brazil
E-mail: luciano_acordi@yahoo.com.br 